\title{
Estimating the permeability of ferrite-polymer composite via a numerical optimization method
}

\begin{abstract}
Estimating the relative complex permeability of each component of samarium-substituted yttrium iron garnet nanoparticles in poly-vinylidene-fluride (Sm-YIG-PVDF) composite samples via a numerical optimization method is performed using a MATLAB program. The optimization is taken as the optimized parameters that yield a minimum sum for the absolute differences between the calculated impedance obtained by using the permeability calculated from Maxwell-Garnett (MG) formula and the measured equivalent one over the entire frequency range named the objective function $(\mathrm{M})$. The guessed (estimated) ranges of the complex permeability are based on the measured values of each component of Sm-YIGPVDF composite samples. The optimized (optimum) impedance values are in very good agreement with the measured ones for each composite and within the estimated ranges. More details on the optimization procedure and the permeability of different composition of the Sm-YIG-PVDF composite materials are illustrated.
\end{abstract}

Keyword: Ferrite-polymer composite; MATLAB; Maxwell Garnett; Permeability 